\title{
Image Segmentation based on FUZZY GLSC Histogram with Dynamic Similarity Discrimination Factor
}

\author{
N. Swathi \\ Gudlavalleru Engineering College \\ Gudlavalleru
}

\author{
K. Ravi Kumar \\ Assistant professor \\ Gudlavalleru Engineering College \\ Gudlavalleru
}

\begin{abstract}
Image processing applications performs image segmentation as pre-processing technique to extract the features for next stage. The application performance depends on image segmentation, to process the foreground or background objects. The image segmentation plays a vital role in computer vision and image processing applications. In spite of having many thresholding techniques in literature they have their own limitations. This paper proposes a new method of thresholding using Gray Level Spatial Correlation (GLSC) histogram with a dynamic similarity discrimination factor ( $\zeta$ ) and Fuzzy logic in deciding the threshold using Shannon's entropy. The similarity discrimination factor $(\zeta)$ is made dynamic by considering the absolute difference between the global and local mean of the image. Calculating the threshold in the Fuzzyfied region makes the segmentation process the most time efficient than the existing methods. Experimental results prove better efficiency $(\eta)$ than the existing methods.
\end{abstract}

The technique out performs in case of low contrast images.

General Terms-Segmentation, histogram, Fuzzy technique.

\section{Keywords}

Entropy, Fuzzyfication, Fuzzyfied image, GLSC histogram, threshold

\section{INTRODUCTION}

Image threshold is a simple and popular technique for image segmentation used in image processing applications to separate foreground or background of an image. For a well defined image its histogram has a deep valley between two peaks. The valley region is the best place to locate the threshold in bimodal histogram images because most of the times, peaks represents the object and background pixels but it is not applicable for all types of images. Many threshold methods are lagging in generosity of their application found in literature [1-3], still it is an area where the research is alive. Otsu proposed discrimination analysis to find threshold, The 2D histogram will produce same threshold for different images having same no of pixels in each intensity level with different pixel distribution. The Gray Level spatial Correlation (GLSC) histogram which takes in to account the spatial correlation of the pixel with its neighborhood differentiates the images with same frequency but different placements. To overcome this problem at 2D histogram, a 3D Gray Level Spatial Correlation (GLSC) histogram with a constant similarity measure 4 is constructed [12].

Let $f(x, y)$ be the gray value of the pixel located at the point $(\mathrm{x}, \mathrm{y})$ in a digital image $\mathrm{F}=\{\mathrm{f}(\mathrm{x}, \mathrm{y}) \mid \mathrm{x} \in\{1,2, \ldots \ldots, \mathrm{Q}\}$, $y \in\{1,2, \ldots \ldots, R\}$ of size $Q \times R$. For the sake of failing in low contrast images[4]. Based on statistical measures like mean, variance, standard deviation Otsu derived threshold. Shannon introduced information theory based on the concept of entropy [5]. Pun used this entropy concept to derive threshold [6]. Kapur et.al. improved the work of Pun [7]. This is extended to Renyi's entropy [8-9] and Tsalli's entropy [10-11]. Yang Xiao et.al. Improved this work by constructing 3D Gray Level Spatial Correlation (GLSC) histogram [12] by considering the local properties of image at constant similarity measure 4 which overcomes the time complexity of 2D histogram approaches [13]. The usage of 3D histogram instead of 2D will result better threshold value, Gray Level Spatial Correlation (GLSC) histogram along with entropic techniques is the recent advancement in this context. In this paper we propose an image segmentation technique based on GLSC histogram with dynamic similarity discrimination factor $(\zeta)$ by considering the local and global characteristics, to improve the method proposed by Yang Xiao. The parent version algorithm using a constant 4 as the similarity measure to construct a $3 \mathrm{D}$ histogram on a $3 \times 3$ window image, does not suits for all types of images. Using Fuzzy technique to extract Fuzzyfied region [14] in image and calculating threshold using Shannon's entropy [15] in this region itself makes the proposed image segmentation technique very time efficient . The redistribution of missing probability amount in floating precisions is made to improve the performance of the proposed method. The parameter efficiency $\eta$ based on misclassification error between segmented image and ground truth image is more than the existing methods.

The remainder of this paper is organized as follows: section 2 explains existing method; section 3 explains proposed method; section 4 illustrates comparative results of proposed, existing methods and parameter efficiency of proposed method over existing methods; section 5 ends up with conclusion.

\section{EXISTING METHOD}

convenience, we denote the set of all gray levels $\{0,1,2 \ldots$. $255\}$ as G. The GLSC histogram is computed as follows. The Fig 2.1 illustrates GLSC histogram with a constant similarity measure $(\zeta)$.

$$
\begin{aligned}
& g(x, y)= \\
& \sum_{i=-\frac{N-1}{2}}^{\frac{N-1}{2}} \sum_{j=-\frac{N-1}{2}}^{\frac{N-1}{2}}(|f(x+i, y+j)-f(x, j)| \leq \zeta)
\end{aligned}
$$

Where 
$(|f(x+i, y+j)-f(x, j)| \leq \zeta)=$

$\begin{cases}1 & \text { if }|f(x+i, y+j)-f(x, y)| \leq \zeta \\ 0 & \text { if } \mid f(x+i, y+j)-f(x, y)>\zeta\end{cases}$

While computing the $\mathrm{g}(\mathrm{x}, \mathrm{y})$, disregarding the $\mathrm{N}$ rows from the top to bottom and $\mathrm{N}$ columns from sides. The pixels gray value, $f(x, y)$ and $g(x, y)$ are used to construct the GLSC histogram using

$\mathrm{h}(\mathrm{k}, \mathrm{m})=\operatorname{Prob}(\mathrm{f}(\mathrm{x}, \mathrm{y})=\mathrm{k}$ and $\mathrm{g}(\mathrm{x}, \mathrm{y})=\mathrm{m})$

Where, $\mathrm{P}$ is gray level correlation probability computed for all pixels with intensity $\mathrm{k} \in \mathrm{G}$ and $\mathrm{m} \in\{1,2, \ldots \ldots \mathrm{N} \times \mathrm{N}\}$ and histogram is normalized [12]. As Yang Xiao said the existing method gives its best results with similarity measure $\zeta=4$ and $\mathrm{N}=3$. Shannon's entropy is used in estimation of threshold value on GLSC histogram instead of 2D histogram with a defined weight for the taken map $\mathrm{N}=3$ and corresponding correlation values from equations 4 and 5 .

$\varphi(\mathrm{t}, \mathrm{N})=\mathrm{H}_{\mathrm{O}}(\mathrm{t}, \mathrm{N})+\mathrm{H}_{\mathrm{B}}(\mathrm{t}, \mathrm{N})$

$\mathrm{t}=\operatorname{Arg} \max \varphi(\mathrm{t}, \mathrm{N})$

Where $\varphi(t, N)$ is the entropic criterion function, $\mathrm{H}_{\mathrm{O}}(\mathrm{t}, \mathrm{N})$ and $\mathrm{H}_{\mathrm{B}}(\mathrm{t}, \mathrm{N})$ are object and background entropies respectively. The normalized histogram is approximated by using the formula.

$\mathrm{h}(\mathrm{k}, \mathrm{m})=$

No of pixels with gray valuek and m pixelsof similar gray valuein $\mathrm{N} \times \mathrm{N}$ neighborhød

No of pixelsin theimage

\section{PROPOSED METHOD}

The existing method proposed by Yang Xiao gives best results at similarity measure 4 but it is not generous to all types of images since it considers only local properties of image. The Gray Level Spatial Correlation (GLSC) histogram which is constructed with a constant similarity measure 4 can be extended by making the similarity measure as a dynamic value. We propose a Gray Level Spatial Correlation (GLSC) histogram with a dynamic similarity discrimination factor $\zeta$ which takes in to account the local and global properties of image. The combined probability of object and background must be unity, but due to floating precisions some amount is missing in both the probabilities. The missing probability is compensated by equally distributing across the probability function $\mathrm{p}(\mathrm{k}, \mathrm{m})$. We propose a Fuzzy technique to extract the Fuzzyfied region in the GLSC histogram of the image. The co-ordinates of Fuzzyfied region is obtained from the proposed method GLSC histogram. Using these co-ordinates the fuzzy functions (s, pi, z) extract the Fuzzyfied region from the GLSC histogram of the image. We use the Shannon's entropy in the estimation of the threshold value. The entropic criterion function is calculated at each and every pixel in the extracted misclassified region or Fuzzyfied region of GLSC histogram. The point at which the entropic criterion function becomes maximum, we choose that point as threshold value. By restricting the threshold calculation to the extracted Fuzzyfied region itself, the time complexity to calculate the threshold value is reduced. By introducing the fuzzy technique, the proposed method becomes very time efficient. The process involved in the proposed method and the corresponding intermediate results at each stage is illustrated as shown in Fig 1.

\subsection{GLSC histogram with dynamic similarity discrimination factor $\zeta$}

We construct Gray Level Spatial Correlation (GLSC) histogram with dynamic similarity discrimination factor $\zeta$ which takes in to account the local and global properties of image. In the existing method Yang Xiao constructed it at constant similarity measure 4 . The dynamic similarity discrimination factor $\zeta$ is calculated for every $\mathrm{N} \times \mathrm{N}$ map of image. The similarity discrimination factor $\zeta$ is made dynamic by considering the absolute difference between the local mean of the considered $\mathrm{N} \times \mathrm{N}$ window and global mean of the image. As the considered local window changes, the local mean changes, consequently the absolute difference between local and global mean changes.

$$
\zeta=\left|M_{g}-M_{1}\right|
$$

The time complexity for calculation of $\zeta$ is less when compared with other statistical measures like variance, covariance and standard deviation. The proposed gray level Spatial Correlation (GLSC) histogram with new $\zeta$ is constructed using equations 1 and 2.Fig.2.a shows 'cameraman.tif' image for which 2D, 3D GLSC with constant similarity measure 4 and 3D GLSC histogram with dynamic similarity discrimination factor $\zeta$ is shown in Fig. 2.b,2.c, $2 . d$ respectively. The $2 \mathrm{D}$ histogram represents two peaks with object and background, the same is represented in GLSC histogram, GLSC histogram with dynamic similarity discrimination factor is represented by considering the local and global properties of image which is used to extract Fuzzyfied region of image and helps Shannon's entropy to attain optimal threshold.

\subsection{Extracting the Fuzzified region of histogram using Fuzzy technique}

Histograms of images with two distinct regions are formed by two peaks separated by a deep valley called bimodal histograms as seen in Fig. 3. In such cases, the threshold value must be located on the valley region. When the image histogram does not exhibit a clear separation, ordinary thresholding techniques might perform poorly. The fuzzy technique provides a new tool to deal with this sort of histograms [16] as shown in Fig. 3. 


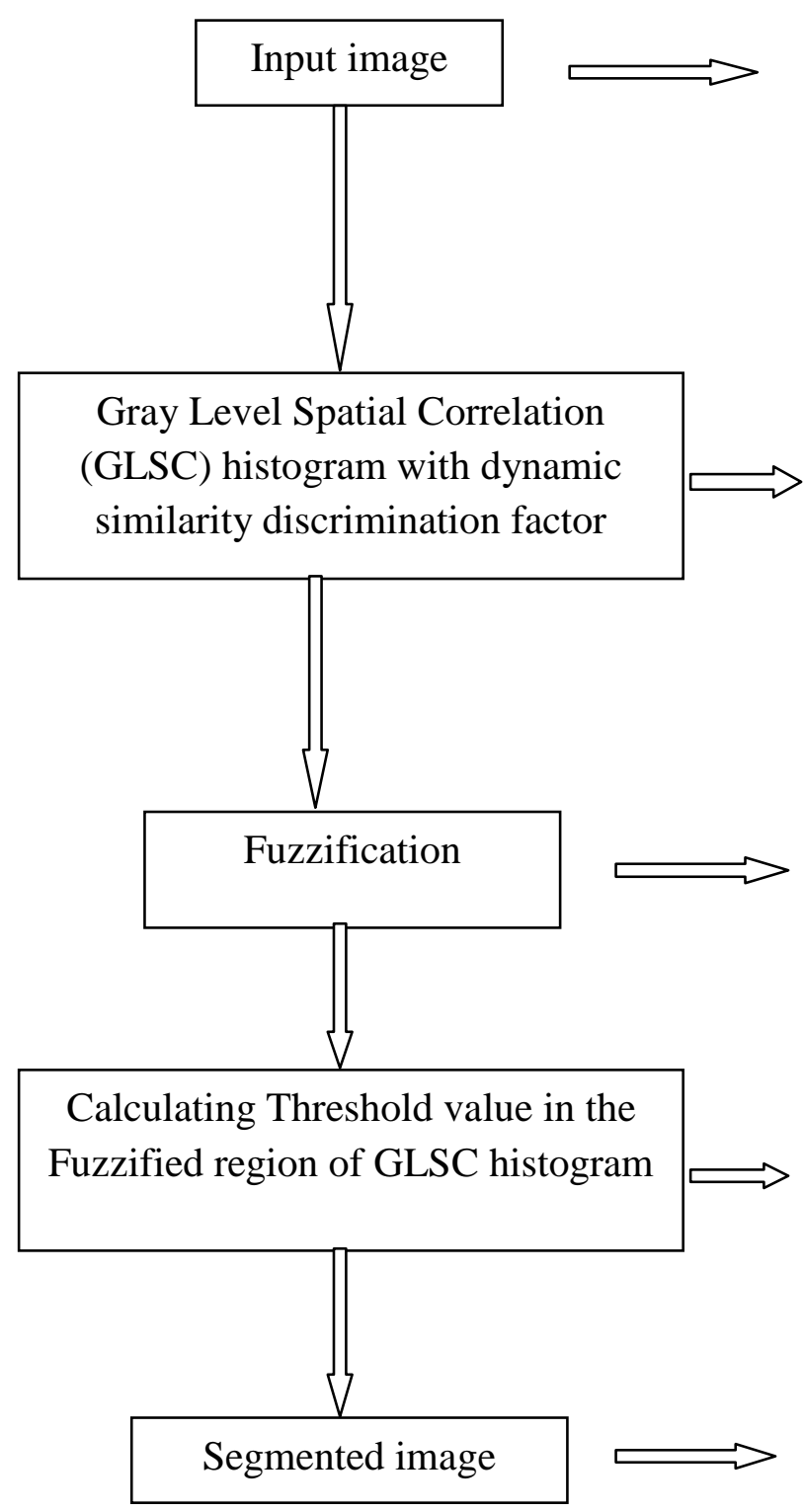

Fig 1: Flowchart of proposed image segmentation technique

The fuzzy functions s, pi, $\mathrm{z}$ functions are used to extract the fuzzy zone of the image histogram and the respective zone is analized for the fuzzyfication process with the help of GLSC histogram and the seed values are obtained from the proposed histogram. The fuzzy functions are derived as

$F(x)=\left\{\begin{array}{ccc}0 & \text { if } & x<A \\ \left(\frac{\mathrm{x}-\mathrm{A}}{\mathrm{C}-\mathrm{A}}\right)^{2} & \text { if } & \mathrm{A}<\mathrm{x}<\mathrm{C} \\ 1 & \text { if } & \mathrm{x}>\mathrm{C}\end{array}\right.$

The equation for $\mathrm{z}$ plot is given by
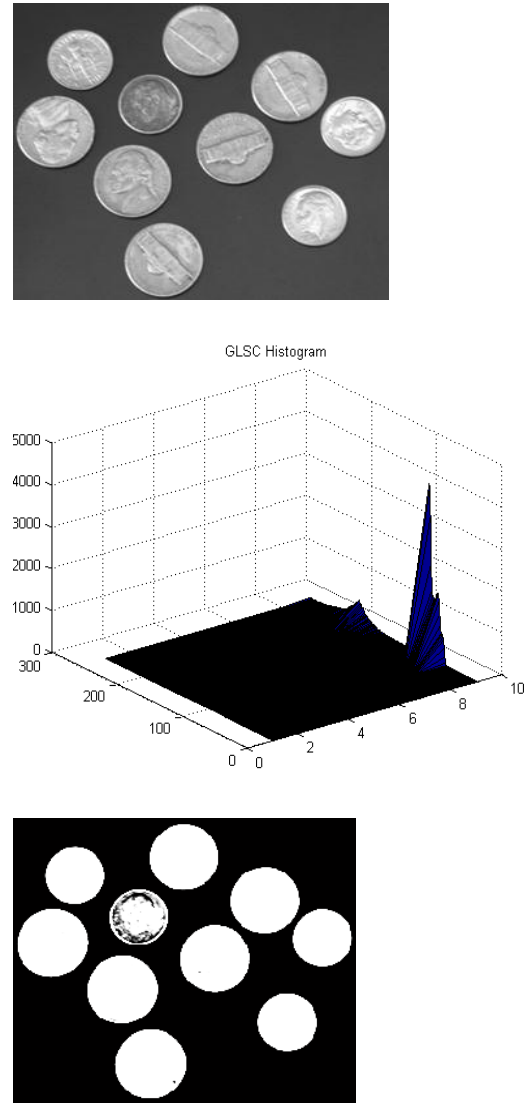

Threshold value $=89$

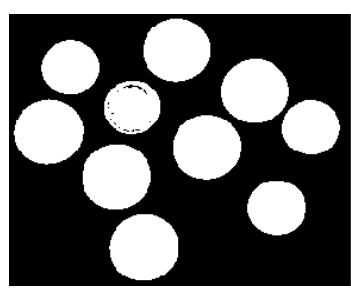

Segmented image

$F(x)=\left\{\begin{array}{ccc}1 & \text { if } & x<A \\ 1-\left(\frac{x-A}{C-A}\right)^{2} & \text { if } & B<x<C \\ 0 & \text { if } & x>C\end{array}\right.$

The pi plot is the combination of $\mathrm{s}$ and $\mathrm{z}$ plots. The coordinates required for these functions are obtained from the constructed GLSC histogram with dynamic similarity discrimination factor $\zeta$. The seed values are obtained as

$\mathrm{B}=\mu($ weighted GLSC histogram $)$

$\mathrm{A}=\mathrm{B}-\sigma($ GLSC histogram $)$

$\mathrm{C}=\mathrm{B}+\sigma($ GLSC histogram $)$ 


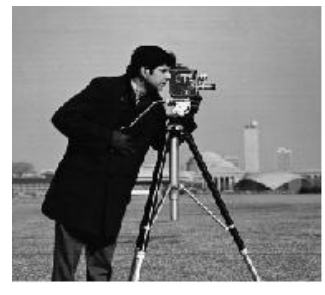

a Cameraman.tif

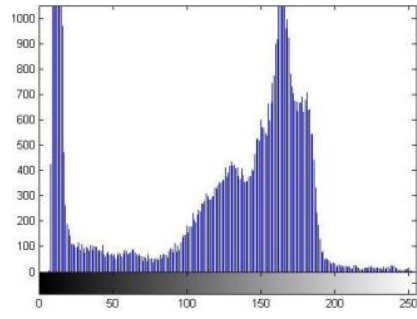

b Conventional 2D histogram

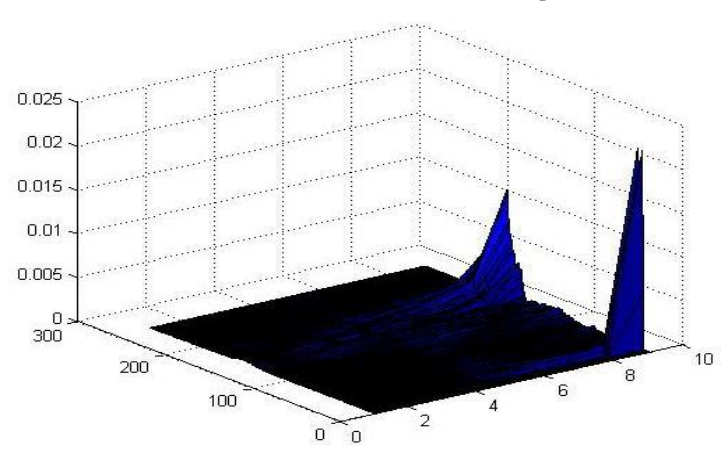

c GLSC histogram with $\zeta=4$

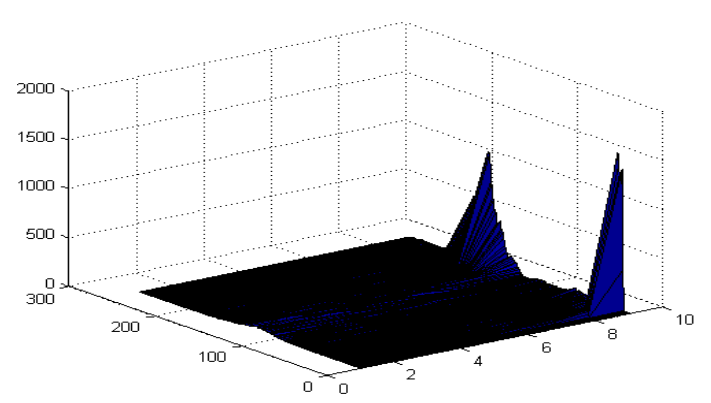

d GLSC histogram with dynamic $\zeta$

Fig 2: Cameraman image, 2D histogram, GLSC and GLSC with dynamic similarity discrimination factor $\zeta$

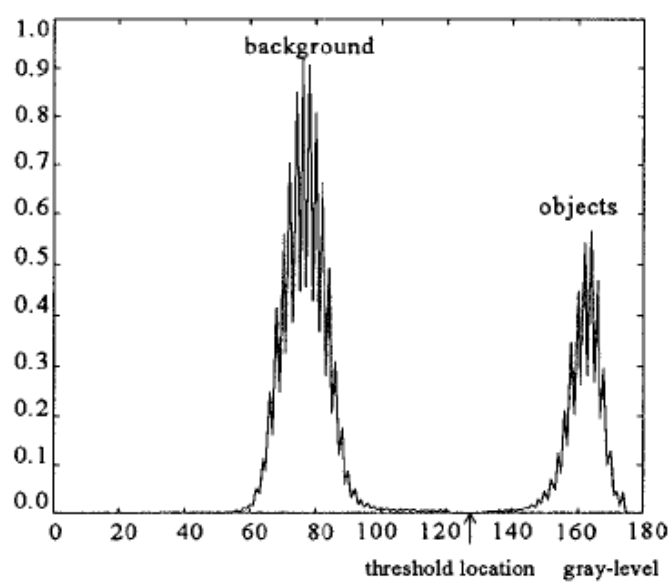

Fig 3: Bimodal image gray level histogram

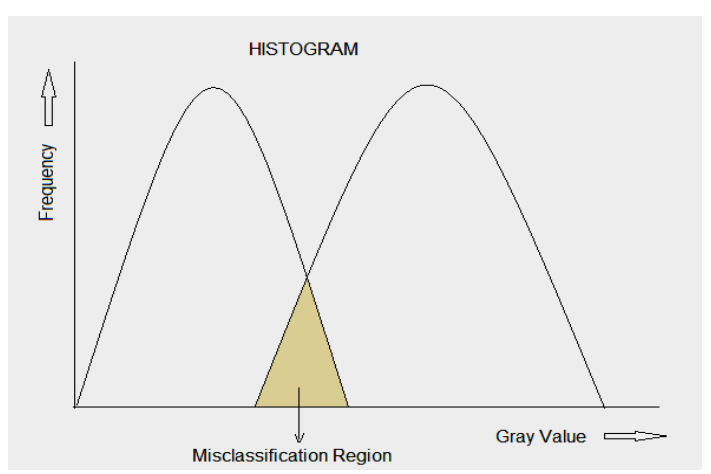

Fig 4: Multimodel image gray level histogram
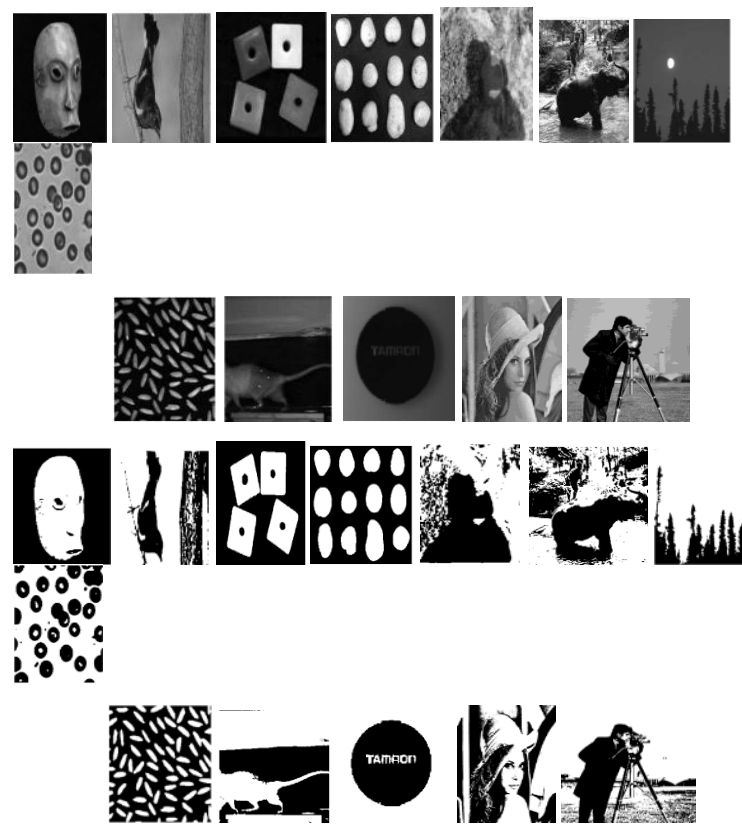

Fig 5: Original image data set and possible ground truth images.

\section{RESULTS AND DISCUSSIONS}

To illustrate the performance of the proposed image segmentation technique we consider 19 images as an image database, varying from uni-modal to multimodal and the corresponding gold standard ground truth images are generated manually to measure a parameter efficiency $\eta$ based on misclassification error using equation(13). Where IMGO and IMGT are ground truth image and segmented images respectively. This efficiency $\eta$ would be 0 for imperfect matching of images and 100 for perfectly matched images. Fig. 5 shows original image set and possible ground truth image set. Efficiency $\eta$ is calculated for each technique on image to dig the performance analysis. More detailed the image set is divided into three different groups from the histogram response as uni-model, bi-modal and multimodal. The performance plots are shown in Fig. 6 and Fig. 7. In each category the proposed method outperforming the existing algorithms. The sample output images are shown in Fig. 8. The mean efficiency confirms quantitative improvement over existing methods.

$$
\eta=\frac{|\mathrm{IMGO} \cap \mathrm{IMG}|}{|\mathrm{IMGO}|} \times 100
$$


Table 1. Performance comparison of Unimodal images

\begin{tabular}{|l|l|l|l|l|l|l|}
\hline S. no & Image & $\begin{array}{c}\text { Proposed } \\
\text { method }\end{array}$ & $\begin{array}{c}\text { Improved } \\
\text { GLSC }\end{array}$ & GLSCH & Otsu & Entropy \\
\hline 1.00 & Scull & 100.00 & 98.69 & 61.67 & 96.50 & 44.04 \\
\hline 12.00 & Roof & 96.12 & 89.91 & 72.88 & 80.52 & 77.48 \\
\hline 13.00 & $\begin{array}{l}\text { Roof } \\
\text { heads }\end{array}$ & 96.16 & 99.24 & 58.38 & 99.33 & 12.41 \\
\hline 14.00 & Sheat & 94.20 & 92.16 & 81.25 & 89.21 & 28.88 \\
\hline
\end{tabular}

Table 2. Performance comparison of bimodal images

\begin{tabular}{|c|c|c|c|c|c|c|}
\hline S.no & Image & $\begin{array}{l}\text { Propose } \\
\text { d } \\
\text { method }\end{array}$ & $\begin{array}{l}\text { Impro } \\
\text { ved } \\
\text { GLSC }\end{array}$ & $\begin{array}{l}\text { GLSC } \\
\mathrm{H}\end{array}$ & Otsu & Entropy \\
\hline 4.00 & $\begin{array}{l}\text { Potato } \\
\text { es }\end{array}$ & 99.69 & 99.52 & 77.32 & 97.39 & 88.70 \\
\hline 7.00 & Trees & 98.75 & 99.60 & 68.14 & 28.29 & 33.27 \\
\hline 8.00 & $\begin{array}{l}\text { Blood } \\
\text { cells }\end{array}$ & 99.00 & 99.82 & 84.21 & 97.90 & 86.85 \\
\hline $\begin{array}{l}16.0 \\
0\end{array}$ & Anshu & 96.58 & 97.67 & 60.29 & 96.92 & 71.04 \\
\hline $\begin{array}{l}17.0 \\
0\end{array}$ & $\begin{array}{l}\text { Wall } \\
\text { flower }\end{array}$ & 99.03 & 96.40 & 75.55 & 95.56 & 64.84 \\
\hline $\begin{array}{l}19.0 \\
0\end{array}$ & Coins & 99.61 & 99.56 & 70.80 & 97.86 & 81.18 \\
\hline
\end{tabular}

Table 3. Performance comparison of multimodal images

\begin{tabular}{|l|l|l|l|l|l|l|}
\hline S.no & Image & Proposed method & Improved GLSC & GLSCH & Otsu & Entropy \\
\hline 2.00 & Bird & 97.21 & 92.23 & 76.36 & 96.13 & 84.85 \\
\hline 3.00 & Blocks & 98.43 & 99.12 & 52.79 & 72.00 & 86.80 \\
\hline 5.00 & Shadow & 94.21 & 97.85 & 94.62 & 98.71 & 98.29 \\
\hline 6.00 & Forest & 97.33 & 93.62 & 74.62 & 90.23 & 100.00 \\
\hline 9.00 & Rice & 98.42 & 98.62 & 61.81 & 96.67 & 81.23 \\
\hline 10.00 & Animal & 96.14 & 97.92 & 78.50 & 52.29 & 84.24 \\
\hline 11.00 & Emblem & 98.66 & 94.22 & 72.51 & 66.46 & 89.47 \\
\hline 15.00 & Lena & 92.92 & 92.00 & 94.06 & 91.44 & 95.02 \\
\hline 18.00 & $\begin{array}{l}\text { Camera } \\
\text { man }\end{array}$ & 96.21 & 93.55 & 91.68 & 91.57 & 84.69 \\
\hline
\end{tabular}




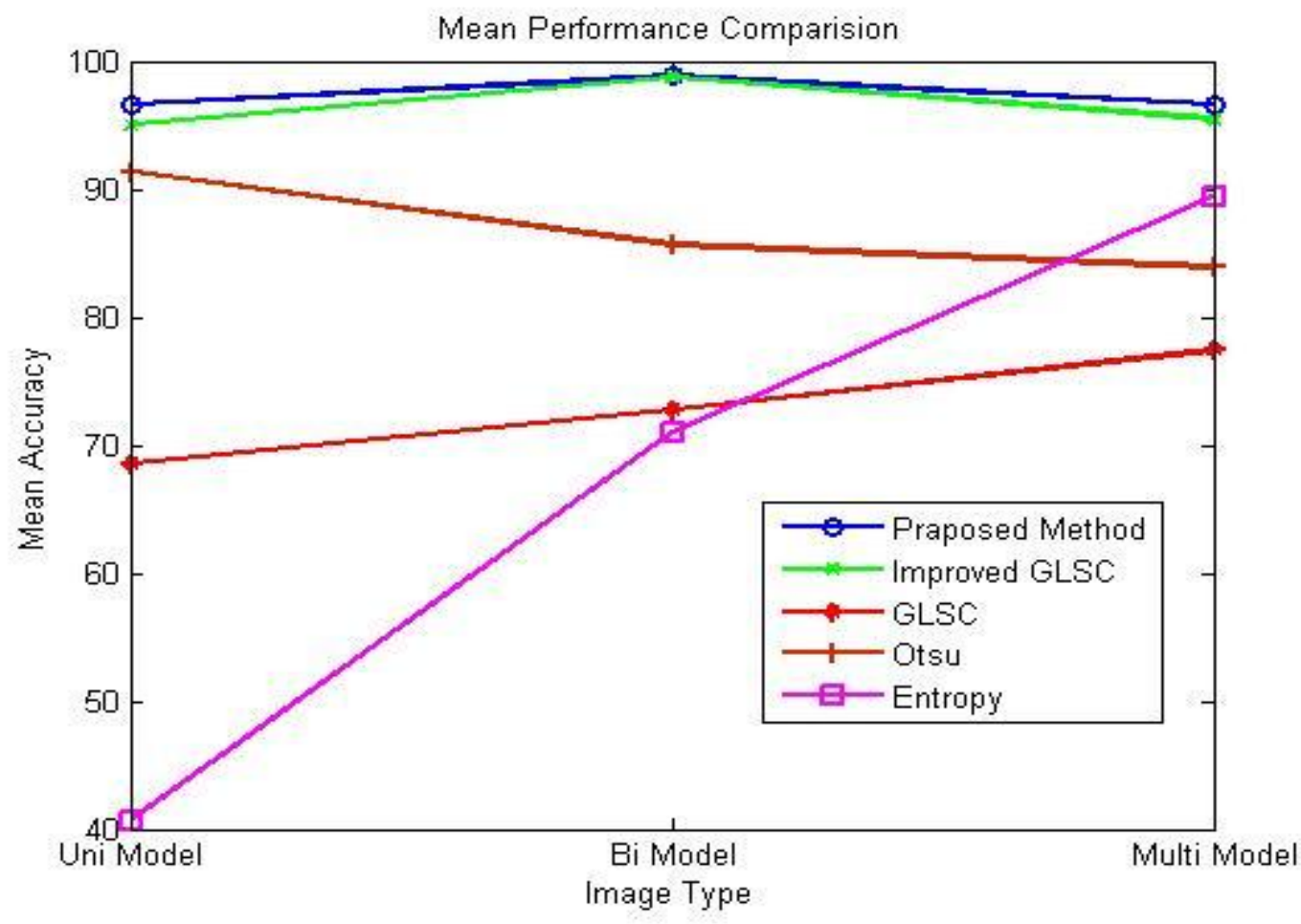

Fig 6: Mean comparison performance.

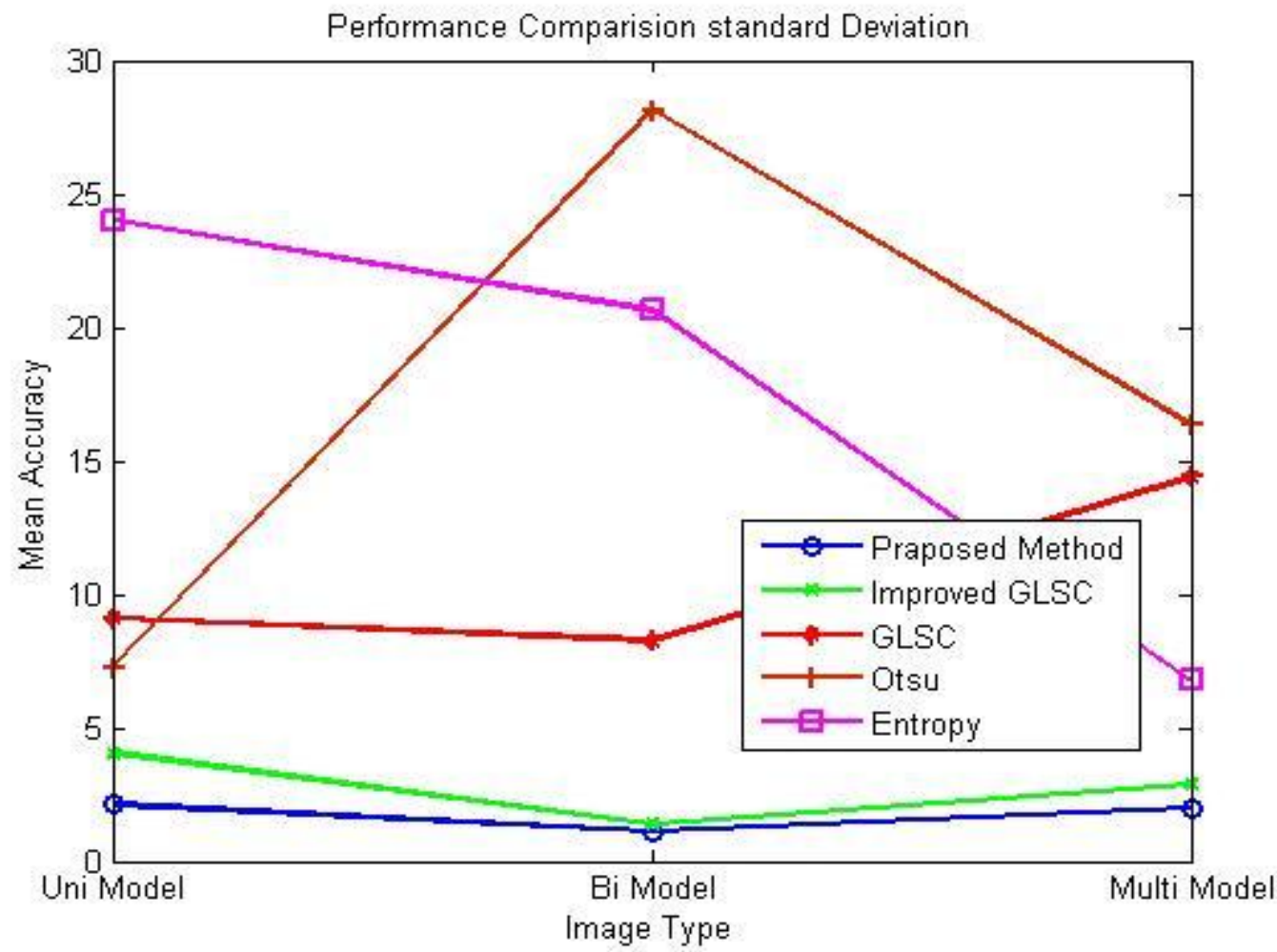

Fig 7: Performace comparison standard deviation. 


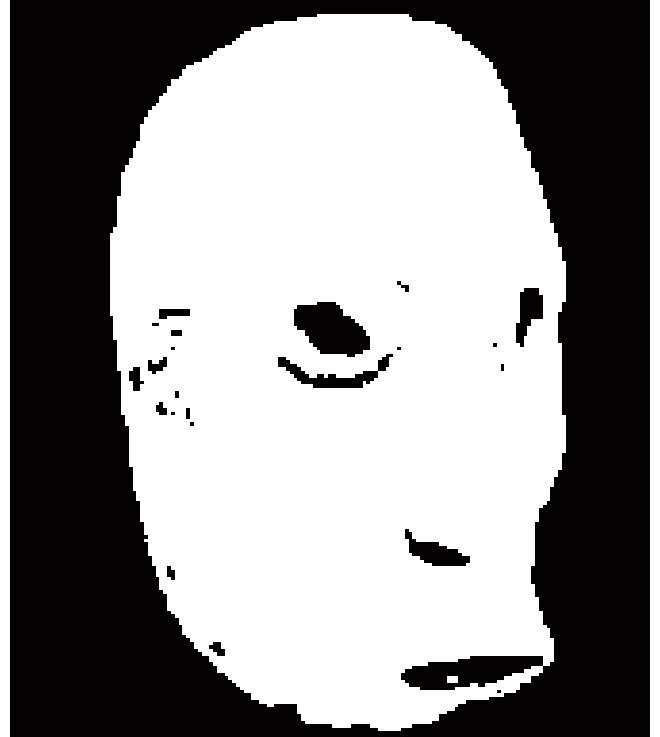

a. Unimodal segmented image

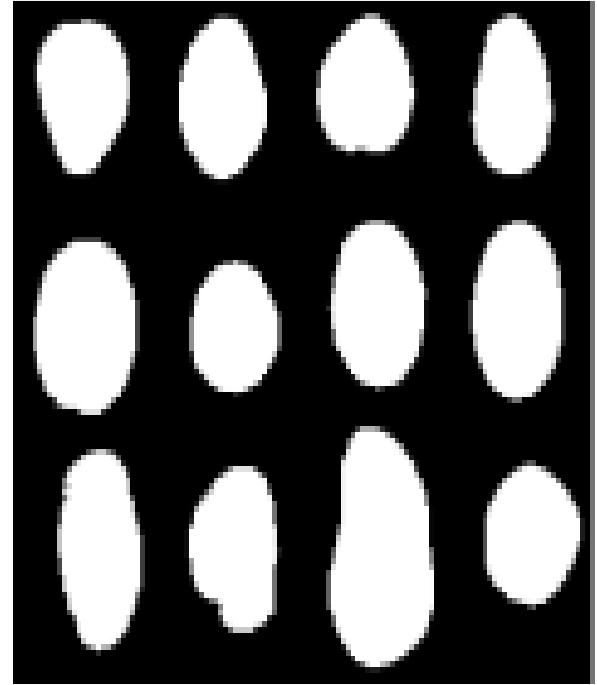

b. Bimodal segmented image

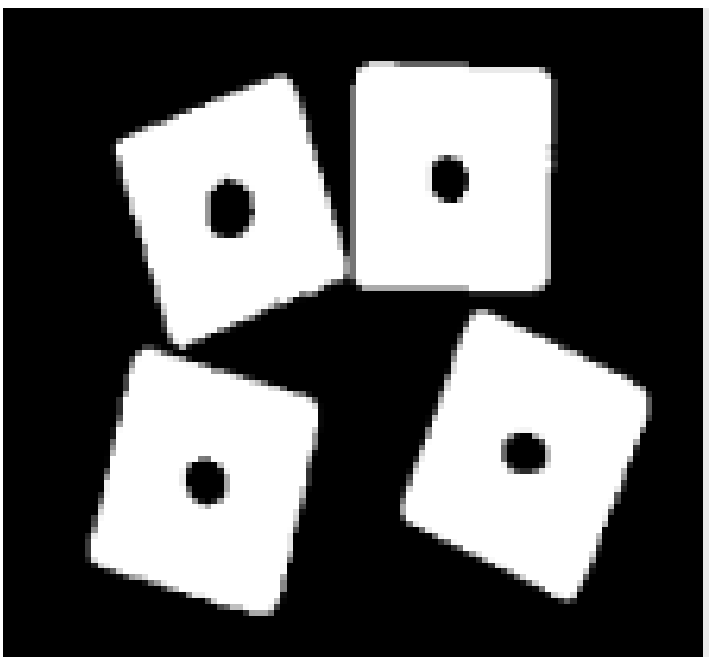

c. Multimodal segmented image

Fig 8: Sample output images of proposed method

\section{CONCLUSION}

A new method of thresholding using Gray Level Spatial Correlation (GLSC) histogram with a dynamic similarity discrimination factor $(\zeta)$ and Fuzzy logic in deciding the threshold using Shannon's entropy was proposed. The similarity discrimination factor $(\zeta)$ was made dynamic. by considering the absolute difference between the global and local mean of the image. The threshold was calculated in the identified Fuzzified region. The seed parameters are obtained from proposed GLSC histogram. Thus, the proposed method becomes less time complex by restricting the calculation of the threshold value only to the extracted Fuzzified region. Experimental results prove better efficiency $(\eta)$ than the existing methods.

\section{REFERENCES}

[1] R. C. Gonzalez and R. E.Woods, Digital Image Processing. Reading, MA: Addison-Wesley, 1993.

[2] M . Sezgin and B. Sankur, "Survey over image thresholding techniques and quantitative $\mathrm{p}$ erformance evalu ation," J.Electron. Imag., vol. 13, no. 1, pp. 146165, Jan. 2004 
[3] N. R. Pal and S. K. Pal, "A review on image segmentation techniques", p attern recog.,vol.26,No. 9, pp.1277-1294,1993.

[4] N. Otsu, "A threshold selection method from gray level histograms," IEEE Trans. Syst., Man, Cybern., vol. SMC-9, pp. 62-66, 1979

[5] C. E.Sh annon,"A mathematical theory of communications”, Bell. Syst.,tech. pp.623-656,J.27,1948

[6] T. Pun, "A new method for gray -level picture thresholding using the entrop y of the histogram," Signal Process., vol. 2, no. 3, pp. 223-237,1980.

[7] J. N. Kap ur, P. K. Sahoo, and A. K. C.Won g, "A new method for graylevel picture thresholding using the entropy of the histogram," Graph. Models Image Process., vol. 29, pp. 273-285, 1985.

[8] P.K. Sahoo, and G. Arora., "A thresholdin g method based on two-dimensional Reny ies entropy ", Pattern Recognit.,

[9] 2004, pp. 1149-1161.

[10] Sahoo, P., Willkins, C., and Yeager, J., "Threshold selection using Reny i"s entropy ", Pattern Recognit., 1997, pp. 71-84.
[11] P.K. Sahoo, and G. Arora, "Image thresholding using twodimensional Tsallis-Havrda-Charvát entropy ", Pattern Recognit. Lett., 2006, pp. 520-528.

[12] Portes de Albuquerque, M., Esquef, I. A., et al., "Image thresholding using Tsallis entropy", Pattern Recognit. Lett.,2004 , pp. 1059-1065.

[13] Yang Xiao, Zhiguo Cao, Tian xu Zhang "Entropic thresholding based on gray level spatial correlation histogram",I EEE trans. 19th international conf., pp. 14,ICPR-2008,

[14] A.S. Abutaleb, "Automatic thresholding of gray -level picture using two-dimensional entropies", Pattern Recognit., 1989 pp.22-32.

[15] A Kaufinnann, Introduction to the Theory ofFuzzy Subsets - Fundamental Theoretical Elements. vol. 1, New York Academic Press, 1975.

[16] E.Pasha, R. Farnoosh, A.Fatemi," Fuzzy Entropy as Cost Function in Image Processing",proceedings of the 2nd IMT -GT Regional conference on Mathematics, Statistics and Applications, Universiti Sains Malaysia, Penang, june 13-15,2006.

[17] [16] Nuno Vieira Lopes, Pedro A. Mogadouro do couto, Humberto Bustince, "Automatic Histogram Threshold Using Fuzzy measures", IEEE Transactions on Image Proceessing,vol.19,No.1,January2010. 